\title{
The Automatic Identification of Hibernating Myocardium
}

\author{
Nicholas M.I. Noble ${ }^{1}$, Derek L.G. Hill ${ }^{1}$, Marcel Breeuwer ${ }^{2}$, and Reza Razavi ${ }^{1}$ \\ 1 Computer Imaging Science Group, Guy’s Hospital, Kings College London, UK \\ $\{$ Nicholas. Noble, Derek.Hill, Reza.Razavi\}@kcl.ac.uk \\ 2 Philips Medical Systems, Medical Imaging Information Technology - Advanced Development \\ Marcel.Breeuwer@philips. com
}

\begin{abstract}
Delayed enhancement imaging is a recently described technique that enables for the first time, the direct observation of areas of myocardium that have scarred following infarction. When this information is combined with information about myocardial contraction, areas that are neither dead, nor contracting can be identified. Such areas will resume contraction following revascularisation (hibernating myocardium). The identification of such areas is consequently of great interest to clinicians. This paper describes how registration can be used to align the images prior to the identification of areas that will benefit from revascularisation. Patient data is used to demonstrate image alignment and image-derived information combination. This is then mapped onto patient-specific $2 \mathrm{D}$ and $3 \mathrm{D}$ representations of the heart.
\end{abstract}

\section{Introduction}

It has recently been observed that gadolinium-based contrast agents have substantially different uptake characteristics in regions of scarred myocardium as opposed to in living areas [1]. Whereas these contrast agents wash into and out of the vascular system of living myocardium in a few heart beats, in areas of scarring, dead cardiomyocytes are replaced by a matrix of collagenous fibres — scarred myocardium — into which contrast agent accumulates 15-20 minutes post-injection. Called delayed enhancement imaging, this technique enables for the first time, the direct non-invasive observation of scarred myocardium. When information about areas of scarred myocardium is combined with knowledge of local myocardial contraction or perfusion, areas of myocardium that are neither dead nor contracting — hibernating myocardium - can be distinguished. Hibernating myocardium has the potential to resume contracting if revascularised [2]. Its location is thus of considerable importance to clinicians planning revascularisation.

Currently, to identify areas of myocardial scar, clinicians use either Positron Emission Tomography (PET), or Single Photon Emission Computed Tomography (SPECT). Scar size measurements using delayed enhancement imaging have been shown to correlate closely with those measured using PET [3]. In addition MR has been shown to systematically detect small sub-endocardial infarcts that were not identified using SPECT [4]. The higher voxel resolution and non-ionising nature of MR make it well suited for the identification of hibernating myocardium. An emergent technology that is competing 
with delayed enhancement is contrast enhanced echocardiography. It remains to be seen which technique emerges as the clinical method of choice.

The identification of hibernating myocardium from MR images can be performed in two ways. The first entails finding areas that demonstrate both perfusion defects in first-pass perfusion images, and an absence of scar in delayed enhancement images. Recently, Breeuwer et al. investigated aligning such images [5]. The second approach involves finding areas of myocardium that exhibit reduced contraction in cine anatomical images, and are not scarred in the delayed enhancement image. To identify such regions the images need to be aligned, this is normally performed mentally by the observer. Differences in the position of the heart due to inter-image motion and inconsistent breath-hold positions confound this already difficult task. This paper will investigate the alignment of cine anatomical images with delayed enhancement images, before identifying areas of hibernating myocardium.

\section{Data}

Short axis ECG triggered steady state free precession images with SENSE factor 2 were obtained in 3 patients undergoing cardiac MRI for the investigation of coronary artery disease. Eight contiguous slices, taken in up to three breath-holds, were imaged with: slice thickness $8-10 \mathrm{~mm}$, field of view $350 \times 344-390 \times 390 \mathrm{~mm}$, acquisition matrix $192 \times 192$ with $120 \%$ phase encode direction sampling, reconstructed to $256 \times 256$ giving a resolution of $1.8 \mathrm{~mm} \times 1.8 \mathrm{~mm}-2 \times 2 \mathrm{~mm}$, with 20 phases in the cardiac cycle, flip angle $50-55^{\circ}$, TE $1.56-1.68 \mathrm{~ms}$ and TR $3.11-3.37 \mathrm{~ms}$. A $0.4 \mathrm{mmol} / \mathrm{kg}$ body weight bolus of gadolinium DPTA was then administered intravenously. Fifteen minutes postinjection, a single end diastolic image was acquired. The images were acquired on a Philips Gyroscan Intera $1.5 \mathrm{~T}$ with master gradients, using a 5 element cardiac synergy coil and vector ECG.

\section{Methods}

Before information about contraction and scarring can be combined, misalignment of the heart between the cine anatomical and delayed enhancement images, due to interscan motion and breath-hold inconsistencies, must be compensated for. Both the delayed enhancement and cine anatomical images are acquired using ECG gating, the delayed enhancement image should consequently be at the same point in the cardiac cycle as the end diastolic cine anatomical image. A rigid registration should hence suffice to align the heart, however, rigid registration of the entire image will only recover differences in bodily position and not those attributable to inconsistent diaphragm position. The registration of a Region Of Interest (ROI) in the delayed enhancement image - defined by manually segmenting the epicardial surface- overcomes this problem. The end diastolic cine anatomical image was thus rigidly registered to the ROI of the delayed enhancement image (both images were preblurred with an isotropic in-plane Gaussian kernel of $2.5 \mathrm{~mm}$ scale). Normalised mutual information [6] was chosen as the similarity measure because of its ability to align areas that include contrast in one image but not the other. The transformation produced by the registration may well result in some degree 

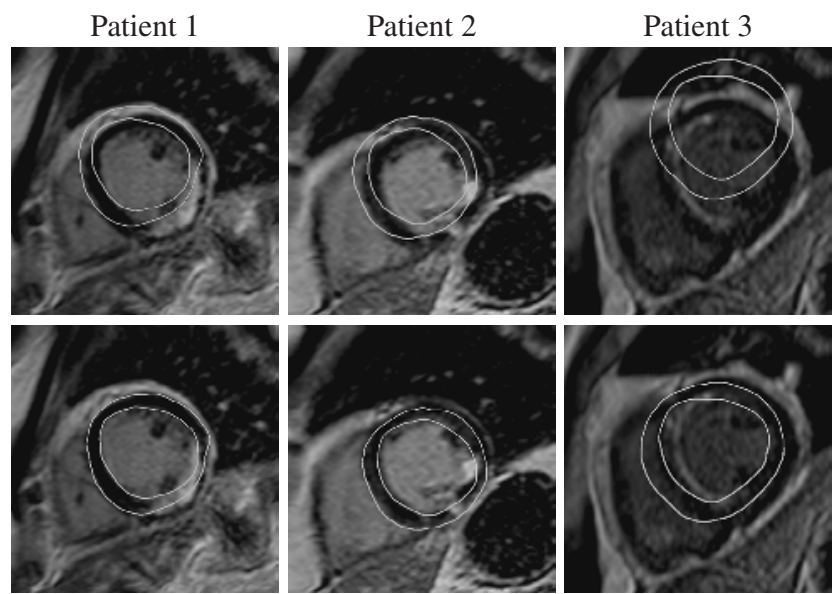

Fig. 1. Manually created end diastolic anatomical contours overlaid onto the delayed enhancement images prior to registration (top row), and following registration (bottom row).

of through-plane motion, because of the massive voxel anisotropy inherent in these images, a smooth 3D contour must hence be created prior to its transformation. Shapebased interpolation [7] of binary volumes produced by segmentation of anatomical end diastolic epicardial and endocardial surfaces provides approximately isotropic voxels. Isotropic Gaussian blurring of the interpolated binary volume with $\sigma=1 \mathrm{~mm}$ followed by the marching cubes algorithm [8], then extracted a smooth 3D surface. Figure 1 shows sections through these contours overlaid on the delayed enhancement image both prior to registration and following registration and transformation. To enable the later assessment of wall thickening, end systolic epicardial and endocardial segmentations were also prepared as described above and transformed by the same transformation. The end systolic image should be correctly aligned with the end diastolic image because it is acquired with the same breath-holds, there is thus no need for alignment of the end diastolic images to the end systolic cine images.

To combine information about contraction and scarring, areas of scarring must first be identified. In the literature, techniques have typically employed intensity thresholding to distinguish areas of scar. Intensity thresholds have been identified manually [5], from manually identified non-scar areas [9], using properties of the histogram [10], using kmeans clustering [11] and also fuzzy k-means clustering [12]. In a comparison of these different thresholding techniques with manually identified areas of scar, fuzzy k-means clustering produced the best results [13]. To demonstrate this technique, the amount of identified scar, expressed as a percentage of the wall thickness - the transmurality of the infarct - was plotted on a bullseye representation of the heart — as is frequently employed in cardiac image analysis packages - Fig.5] first column. However, as can be seen from the adjacent plots of transmurality following manual identification of areas of scar, fuzzy k-means clustering mis-classifies large amounts of myocardium. 

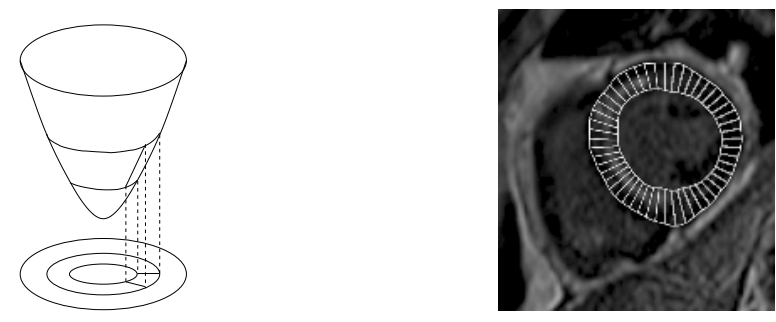

Fig. 2. Illustration of how the three-dimensional location of myocardial segments are mapped onto the 2D bullseye plot (left). Parameters for each segment of the bullseye plot were measured in-slice at equi-spaced angular locations about the in-slice centroid of the delayed enhancement endocardial contour (right).

A bullseye plot is a series of contiguous concentric rings, segments of each ring are colour coded according to the parameter calculated for corresponding segments of myocardium. Each of the concentric rings in the plot represents information originating from a particular slice, the slice nearest the apex representing the innermost ring, and the outermost ring information from the slice nearest the base - Fig. 2. The in-plane angular location of the information determines the angular location at which it is positioned in the bullseye plot. In this case, parameters for each segment of the bullseye plot were measured in-slice at equi-spaced angular locations about the in-slice centroid of the delayed enhancement endocardial surface — Fig. 2

However, thresholding by itself as a means of identifying scar tissue is unsatisfactory in two respects. Firstly, the probability density functions of healthy myocardium and enhanced areas often overlap. In the absence of any spatial continuity constraints, this leads to voxel mis-classification. Secondly, microvascular obstruction in areas of scarring can sometimes totally prevent the entrance of any contrast agent whatsoever, resulting in areas of scar with 'dark cores' [14]. The sole exception to the employment of thresholding for the purpose of scar identification is O'Donnell et al.'s work [15], they employ support vector machines [1617] to identify the hyper-surface in parameter space that separates scarred from unscarred tissue, this technique requires a large amount of training data which prevented our investigation of it. Although not explicitly stated in the paper, this technique may be able to correctly classify dark core areas.

Given the high incidence of mis-classification when using fuzzy k-means clustering, the general flaws associated with thresholding techniques, and our inability to assess O'Donnell's technique due to the number of data sets required for training, areas of scar were identified manually and the transmurality plotted on a bullseye - Fig. 5, second column. The percentage wall thickening (a metric routinely employed in echocardiography) was then calculated from the transformed end diastolic and end systolic contours, and mapped onto bullseyes -Fig.5 third column. Because the contours have been aligned prior to being mapped onto the bullseye plots, for the purposes of data combination, it will be sufficient to combine the information associated with the segments of the bullseye plots. 

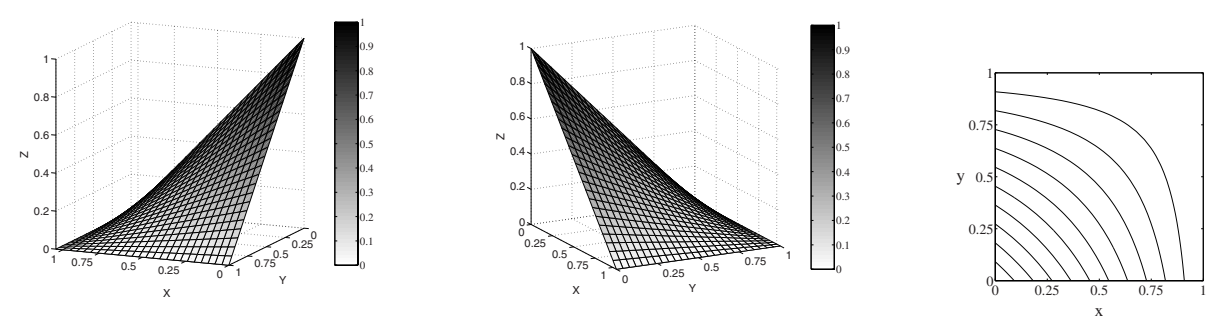

Fig. 3. Two different views of the revascularisation candidacy surface as described by $z=(1-$ $x)(1-y)$ where $x$ and $y$ represent the transmurality and wall thickening values (left and middle) and a contour map of the surface (right).

To combine the information from the two bullseye plots, a function of the wall thickening $(x)$ and transmurality $(y)$ needs to be defined. This function should be maximum in areas of low thickening and low scarring, indicating the likely location of hibernating myocardium — candidate areas for revascularisation. The function should in addition be minimum in areas of maximal thickening and also in areas of maximal transmurality - areas, the revascularisation of which, will not benefit the patient. One such candidacy function can be described by $z=(1-x)(1-y)$, Fig. 3 . This function was used to combine the wall thickening and transmurality information on bullseye plots for each patient -Fig. 5, fourth column. It should be noted that although this function has the desired features as described above, its behaviour away from these points is a somewhat arbitrary estimation of the benefit associated with the revascularisation of such areas. The true revascularisation candidacy function, can only be determined experimentally by the assessment of both pre- and post-revascularisation images.

Although the bullseye plot permits two-dimensional observation of the location of three-dimensional data, it is somewhat abstract. An easier to interpret representation would be if the combined information were visualised on a 3D representation of the myocardium. To thus represent the combined wall thickening and transmurality information, a coordinate system that can be used to translate between the bullseye plot and the surface must be defined. Segments in the bullseye plot can be easily identified using a polar representation, on the surface this corresponds to a $(z, \theta)$ coordinate system - as opposed to an $(r, \theta)$ system on the bullseye plot. The location of the centroid of any of the 3D surface's facets on the bullseye can be identified from its z-position and its theta value calculated with respect to its in-slice centroid. This is a continuous coordinate system and the resulting location in the bullseye plot may consequently be at a noninteger position. To enable interpolation of intermediate values from the bullseye plot, it was unwrapped (see Fig. 44). Cubic b-spline interpolation - wrapped around at the image edges to account for radial continuity - then enabled smooth $C 2$ interpolation of the bullseye plot. Examples of the bullseye plots for transmurality, wall thickening and the combined function are shown mapped onto prepared segmentations of the delayed enhancement epicardial surface in Fig.6. 

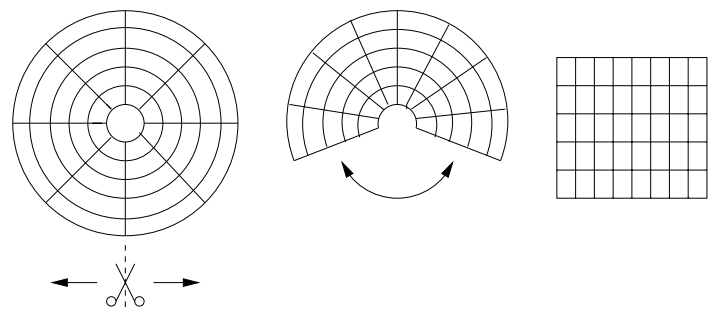

Fig. 4. The unwrapping of the bullseye plot into a rectangular image.

\section{Results}

The contour overlays in Fig.1 1 clearly demonstrate that for patients 1 and 2, the registration has aligned the hearts very well. For patient 3 however, there is a substantial registration error. It would seem that the registration algorithm has aligned the enhanced region with the blood pool in the cine anatomical image. As can also be seen from Fig.1. patient 3's delayed enhancement image is of poor quality when compared to patient 1 and 2's images. Experimental investigation of the mis-registration concluded that it was due to poor contrast because of inappropriate TI selection during imaging [13]. Despite this poor result, it was decided to continue with the analysis of patient 3 for the purpose of illustrating the technique.

Figure 5 shows bullseye plots of transmurality, wall thickening and candidate areas for revascularisation for all three patients. It can be seen that patient 1 has a sizable amount of scar tissue in the basal and mid anterior segments of the heart, a corresponding area of reduced thickening can be seen in the wall thickening plot. The combined plot does not indicate any substantial areas with high revascularisation candidacy, although a small region may be seen in the basal septal areas. This is nicely illustrated in Fig.6 where for the first two positions, one can clearly see the reduced wall thickening in areas of scar.

Patient 2 has two small areas of scar in the basal anterior segments, the wall thickening bullseye plot shows reduced thickening in this area and also in the septal segments. When combined, it can be seen that there is a large candidate area for revascularisation in the basal and mid septal segments. This is also seen in Fig.6.

The bullseye plot for patient 3 -Fig.5 demonstrates a large septal area of scarring in the mid slices, the transmurality is around $50 \%$ for most of the scarred tissue. Reduced wall thickening is correspondingly seen in the wall thickening plot. The combined plot is patchy, not distinctly indicating any large areas suitable for revascularisation, however the septal side of the heart does have seem to have slightly higher candidacy values.

\section{Discussion and Conclusion}

This paper has addressed the task of automatically combining information from cine anatomical and delayed enhancement images and, to the author's knowledge, is the first paper to attempt this. Rigid registration using normalised mutual information of a 

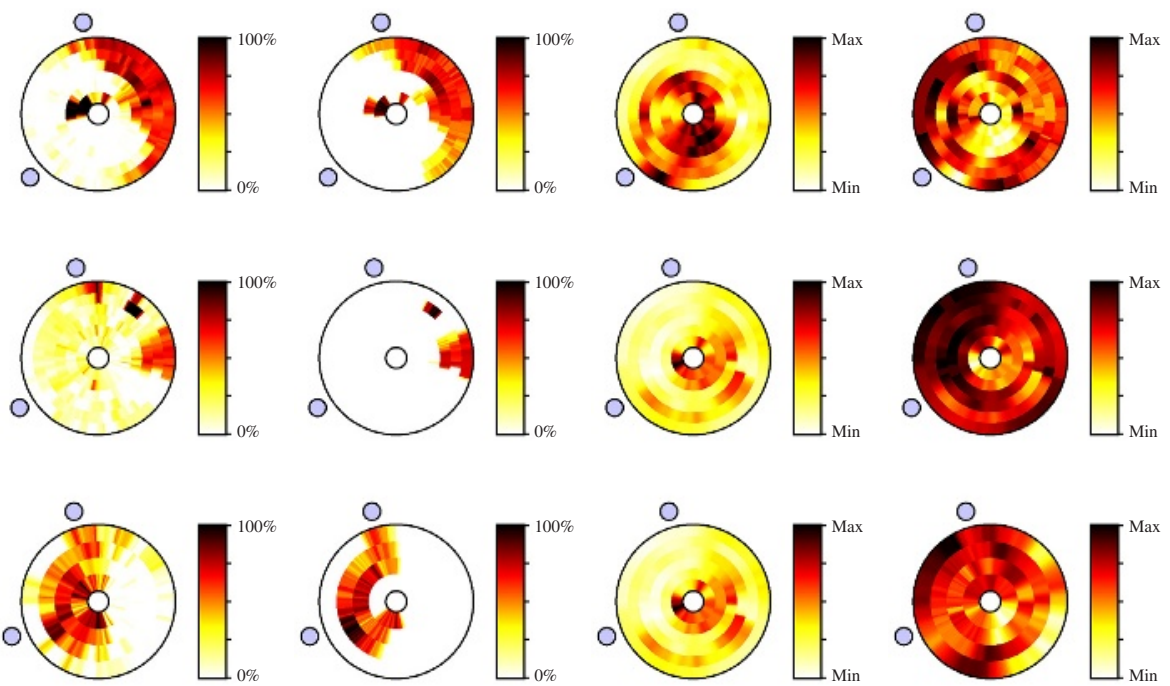

Fig. 5. Bullseye plots showing; transmurality determined using fuzzy k-means clustering [12] (first column), transmurality determined manually (second column), wall thickening (third column), and candidate areas for revascularisation (fourth column), for patients 1, 2 and 3 (top, middle and bottom rows). Dots indicate the location of the conjunction of the left and right ventricles.
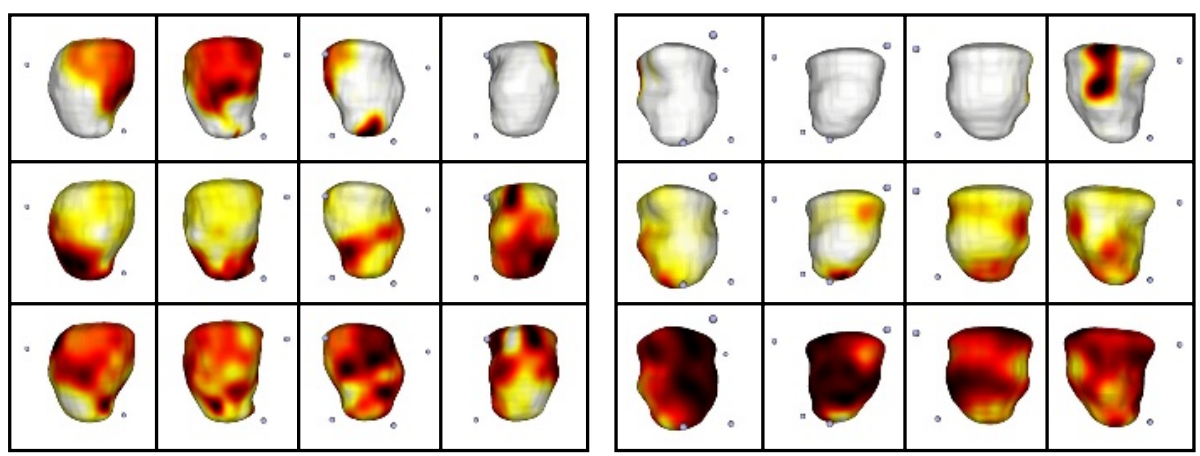

Fig. 6. Mappings of transmurality (top row), wall thickening (middle row) and candidate areas for revascularisation (bottom row) for patients 1 (left) and 2 (right). Spheres indicate the location of the conjunction of the left and right ventricles.

region of interest was shown be to sufficient to compensate for both inter-scan patient movement and inconsistent breath-hold positions. Following registration, the location of manually identified areas of scarring was then combined with manually determined wall thickening information using a combination function. Although at this stage the 
combination function is rather arbitrary, it enables candidate areas for revascularisation to be highlighted for the first time. The results of the information combination were shown on both $2 \mathrm{D}$ bullseye representations of the heart, and on $3 \mathrm{D}$ representations of the myocardial surface. Future work required to make this technique clinically employable would be the empirical identification of a revascularisation candidacy function, this will require a substantial set of both pre- and post-revascularisation images. Following this, the technique could be validated by comparison with results derived from PET-the current gold standard.

Acknowledgements. We are grateful to Philips Medical Systems Nederland B.V. Medical Imaging Information Technology - Advanced Development for funding this work, and to Professor Hawkes and all those at the Computer Imaging Sciences Group for their assistance.

\section{References}

1. R.J. Kim, D.S. Fieno, T.B. Parrish, K. Harris, E. Chen, O. Simonetti, Bundy J., J.P. Finn, F.J. Klocke, and R.M. Judd. Relationship of MRI Delayed Contrast Enhancement to Irreversible Injury, Infarct age, and Contractile Function. Circulation, 100:1992-2002, 1999.

2. S. Rahimtoola. The Hibernating Myocardium. Am. Heart J., 117(1):211-230, 1989.

3. C. Klein, S.G. Nekolla, F.M. Bengel, M. Momose, A. Sammer, F. Haas, B. Schnackenburg, W. Wolfram Delius, H. Mudra, D. Wolfram, and M. Schwaiger. Assessment of myocardial viability with contrast enhanced magnetic resonance imaging: comparison with positron emission tomography. Circulation, 105(2):162-167, 2002.

4. A. Wagner, H. Mahrholdt, T.A. Holly, M.D. Elliott, M. Regenfus, M. Parker, F. Klocke, R.O. Bonow, R.J. Kim, and R.M. Judd. Contrast-enhanced mri and routine single photon emission computed tomography (SPECT) perfusion imaging for detection of subendocardial myocardial infarcts: an imaging study. Lancet, 361:374-379, 2003.

5. M. Breeuwer, R. Muthupillai, S. Flamm, E. Nagel, I. Paetsch, J. Ridgeway, and S. Plein. Combining first-pass and late-enhancement myocardial perfusion analysis. In Proc. Society of Cardiovascular Magentic Resonance, 2003.

6. C. Studholme, D.L.G. Hill, and D.J. Hawkes. An Overlap Invariant Entropy Measure of 3D Medical Image Alignment. Pattern Recognition, 32:71-86, 1999.

7. G.T. Herman, J. Zheng, and C.A. Bucholtz. Shape-Based Interpolation. IEEE Comput. Graph. Appl., pages 69-79, 1992.

8. W.E. Lorensen and H.E. Cline. Marching Cubes: A High Resolution 3D Surface Reconstruction Algorithm. Comput. Graph., 21(4):163-169, 1987.

9. B.L. Gerber, C.E. Rochitte, D.A. Bluemke, J.A. Melin, P. Crosille, L.C. Becker, and J.A.C. Lima. Relation between Gd-DTPA contrast enhancement and regional inotropic response in the periphery and center of myocardial infarction. Circulation, 104:998-1004, 2001.

10. A. Kolipake, G.P. Chatzimavroudis, R.D. White, and R.M. Setser. Segmentation of non-viable myocardium in delayed enhancement magnetic resonance images. In ISMRM, 2003.

11. P. Madhav, V. Mai, M. Zhang, and Q. Chen. An Automated Segmentation Method for Assesing Myocardial Infarct Size Using K-Means Algorithm. In ISMRM, 2003.

12. V. Positano, A. Pingitore, M.F. Santarelli, M. Lombardi, L. Landini, and A. Benassi. Quantitative 3D assessment of myocardial viability with MRI delayed contrast enhancement. In Computers in Cardiology, Thessalonika, Greece, 2003. 
13. N.M.I. Noble. Information alignment and extraction from cardiac magnetic resonance images. $\mathrm{PhD}$ thesis, University of London, 2004. Publication pending.

14. A.S. John and D.J. Pennell. Cardiovascular magnetic resonance imaging of dysfunctional myocardial tissue in ischemic heart disease. Heart Metab., 20:19-22, 2003.

15. T. O'Donnell, N. Xu, R. Setser, and R.D. White. Semi automatic segmentation of non-viable cardiac tissue using cine and delayed enhancement magnetic resonance images. In Proc. SPIE medical imaging, volume 5031, pages 242-251, 2003.

16. C. Burgess. A tutorial on support vector machines for pattern recognition. Data Mining and Knowledge Discovery, 2(2):1-43, 1998.

17. N. Christiani. An introduction to support vector machines and other kernal-based learning methods. Cambridge university press, 2000. 